\title{
Characterizing entanglement using quantum discord over state extensions
}

\author{
Chunhe Xiong, ${ }^{1,2,3, *}$ Sunho Kim, ${ }^{4, \dagger}$ Daowen Qiu, ${ }^{1,5, \ddagger}$ Asutosh Kumar, ${ }^{6,7,8, \S}$ and Junde $\mathrm{Wu}^{9,}$ ฯ \\ ${ }^{1}$ Institute of Quantum Computing and Computer Theory, \\ School of Computer Science and Engineering, Sun Yat-sen University, Guangzhou 510006, China \\ ${ }^{2}$ Interdisciplinary Center for Quantum Information, Department of Physics, Zhejiang University, Hangzhou 310027, China \\ ${ }^{3}$ School of Computer and Computing Science, Zhejiang University City College, Hangzhou 310015, China \\ ${ }^{4}$ School of Mathematical Sciences, Harbin Engineering University, Harbin 150001, China \\ ${ }^{5}$ Instituto de Telecomunicaçốes, Departamento de Matemática, \\ Instituto Superior Técnico, Av. Rovisco Pais 1049-001, Lisbon, Portugal \\ ${ }^{6}$ P.G. Department of Physics, Gaya College, Magadh University, Rampur, Gaya 823001, India \\ ${ }^{7}$ Harish-Chandra Research Institute, HBNI, Chhatnag Road, Jhunsi, Allahabad 211019, India \\ ${ }^{8}$ Vaidic and Modern Physics Research Centre, Bhagal Bhim, Bhinmal, Jalore 343029, India \\ ${ }^{9}$ School of Mathematical Sciences, Zhejiang University, Hangzhou 310027, PR China
}

\begin{abstract}
We propose a framework to characterize entanglement with quantum discord, both asymmetric and symmetric, over state extensions. In particular, we show that the minimal Bures distance of discord over state extensions is equivalent to Bures distance of entanglement. This equivalence places quantum discord at a more primitive position than entanglement conceptually in the sense that entanglement can be interpreted as an irreducible part of discord over all state extensions. Based on this equivalence, we also offer an operational meaning of Bures distance of entanglement by connecting it to quantum state discriminations. Moreover, for the relative entropy part, we prove that the entanglement measure introduced by Devi and and Rajagopal [A. R. U. Devi and A. K. Rajagopal, Phys. Rev. Lett. 100, 140502 (2008)] is actually equivalent to the relative entropy of entanglement. We also provide several quantifications of entanglement based on discord measures.
\end{abstract}

\section{INTRODUCTION}

Quantum correlations [1, 2] are defined from different viewpoints and they, in turn, are expected to offer different advantages. Hence, the characterization and quantification of quantum correlations is instrumental in exploring and exploiting the quantum phenomena. The remarkable advantages that quantum correlations offer make quantum information theory more powerful than classical theory. Entanglement [1] is an important quantum resource which plays a crucial role in quantum information processing, quantum algorithms, quantum computation and cryptography [3]. The notion of quantum correlations and resources beyond entanglement [2, 46] such as quantum discord [2, 7-10], quantum coherence $[6,11-13]$, etc. are also very prominent and useful in quantum information theory. For instance, quantum discord is the genuine resource in the DQC1 algorithm [14, 15].

Among several quantum correlations, entanglement and quantum discord are significant, and are usually regarded as very distinct in nature. While entanglement belongs to the entanglement-separability paradigm, quantum discord belongs to the information-theoretic paradigm. Nevertheless, entanglement and quantum discord have both essential similarities and significant differences. Several remarkable investigations [16-28] relating to entanglement and discord have been studied in recent years. Especially, the correspondence between classical states versus separable states [29], the

\footnotetext{
*xiongch@zucc.edu.cn

$\dagger$ kimsunho81@ @rbeu.edu.cn

‡issqdw@mail.sysu.edu.cn

$\S$ asutoshk.phys@gmail.com

『wjd@zju.edu.cn
}

characterization and quantification of entanglement with the generalized information-theoretic measure [30], the minimal quantum discord of bipartite state over state extensions [31] and the minimal correlated coherence over symmetric state extensions [32,33] are of special interest.

It should be noted that the framework that quantifies entanglement with quantum discord or coherence over state extensions is quite different from the existing entanglement measures such as entanglement of formation and entanglement cost [34], distillable entanglement [35], relative entropy of entanglement and Bures distance of entanglement [36, 37], robustness of entanglement [38], and squashed entanglement $[39,40]$, which are mostly based on operational meaning, information principles, and mathematics. Thus far, the interrelationship between these two different categories of entanglement measures is not clear.

In this paper, we study characterization of entanglement using quantum discord over state extensions. For what do we mean by "over state extensions", see [41]. Our work is different from and generalisation of previous works [30-32]. Authors in Ref. [30] introduced quantumness of correlations as an entanglement measure. They proved that for a bipartite state $\rho_{a b}$, the relative entropy of asymmetric discord over all extended states is a candidate of entanglement measure, that is, this quantification is faithful for separable states and non-increasing under local operations. It also turns out to be an upper bound to the relative entropy of entanglement. On the other hand, it was shown in Ref. [31] that the minimal discord over all extended states is also a valid entanglement measure. These two investigations revealed the potential relation between entanglement and quantum discord over state extensions. Also, the conservation law for distributed entanglement and quantum discord [19] hints at some kind of fundamental relation between them. However, a general formal- 
ism relating entanglement and discord (quantum correlations regarded as very distinctive in nature) has been elusive. Our study provides a natural connection between the two via state extensions. The main findings of our work can be summarised as follows.

(i) For Bures distance [42, 43], we prove that the minimal Bures distance of discord over state extensions is equivalent to Bures distance of entanglement [37, 44, 45]. Moreover, Bures distance of entanglement was proved to be equal to its corresponding convex roof [45], which plays a key role in our study. In fact, we prove the equivalence by showing that the minimal Bures distance of discord over state extensions is bounded by the Bures distance of entanglement and its convex roof.

Bures distance of entanglement is defined from the geometric viewpoint whose operational meaning is not very clear. A correspondence, however, between Bures distance of discord and quantum state discriminations has been established in Ref. [46]. Based on this correspondence and the equivalence between Bures distance of entanglement and discord over state extensions, we offer an operational meaning of $\mathrm{Bu}$ res distance of entanglement by linking it to quantum state discriminations.

(ii) We propose a framework to characterize entanglement using quantum discord over state extensions. Actually, for a generalized discord measure including entropic discord [7, 8], geometric discord [47], measurement-induced geometric discord [48], correlated coherence [32] and geometric correlated partial coherence [49], we prove that the minimal discord over state extensions is a candidate of an entanglement measure. This provides an alternative perspective to understand entanglement from the viewpoint of quantum discord. Results in previous studies [30-32] are special cases of our framework.

(iii) We show that quantification of entanglement proposed in Ref. [30] is actually equivalent to the relative entropy of entanglement [37], which is a well-studied entanglement measure.

This paper is structured as follows. In Sec. II, we recall various concepts prerequisite for our study. We discuss characterization of entanglement using asymmetric and symmetric quantum discord in Sec. III and Sec. IV respectively. We give an operational meaning of the Bures distance of entanglement in Sec. III C. We conclude our findings in Sec. V. Proofs of some theorems and symbols with their meanings are presented in Sec. VI (Appendix) at the end of the bibliography.

\section{PRELIMINARIES}

In this section, we briefly recall various concepts which are prerequisite for our study.

\section{A. Separable states}

Let $\mathcal{H}=\mathcal{H}_{a} \otimes \mathcal{H}_{b}$ be the composite Hilbert space of a bipartite system, and $D(\mathcal{H})$ be the set of density matrices on $\mathcal{H}$. A quantum state $\rho_{a b} \in D(\mathcal{H})$ shared between two parties $a$ and $b$ is called separable if it can be represented as

$$
\rho_{a b}=\sum_{i} p_{i} \rho_{a}^{i} \otimes \rho_{b}^{i},
$$

where $p_{i} \geq 0, \sum_{i} p_{i}=1$ and $\rho_{a}^{i}, \rho_{b}^{i}$ are local states for parties $a$ and $b$. Otherwise, it is called entangled. We denote the set of separable states by $\mathcal{S}$. Moreover, a bipartite separable state $\rho_{a b}$ is called classical-quantum $(\mathcal{C Q})$ if it can be written as $\rho_{a b}=\sum_{i} p_{i}|i\rangle_{a}\langle i| \otimes \rho_{b}^{i}$, and classical-classical $(\mathcal{C C})$ if $\rho_{a b}=\sum_{i, j} p_{i j}|i\rangle_{a}\langle i|\otimes| j\rangle_{b}\langle j|$, where $\{|i\rangle\},\{|j\rangle\}$ are two set of orthogonal pure states.

\section{B. Relative entropy}

Relative entropy is defined as $S(\rho \| \sigma):=\operatorname{tr}[\rho(\log \rho-$ $\log \sigma)$ ] [3]. Throughout, unless stated otherwise, the base of the $\log$ should be taken 2. For any classical-quantum state $\sigma_{a b}^{(c q)}=\sum_{j} p_{j}|j\rangle_{a}\langle j| \otimes \sigma_{b}^{j}$, it is easy to verity that $\operatorname{tr}\left(\rho_{a b} \log \sigma_{a b}^{(c q)}\right)=\operatorname{tr}\left[\Pi_{a}\left(\rho_{a b}\right) \log \sigma_{a b}^{(c q)}\right]$, where $\Pi_{a}\left(\rho_{a b}\right)=$ $\sum_{i}\left(|i\rangle_{a}\langle i| \otimes I_{b}\right) \rho_{a b}\left(|i\rangle_{a}\langle i| \otimes I_{b}\right)$. As a result, one has

$$
\begin{aligned}
& S\left(\rho_{a b} \| \sigma_{a b}^{(c q)}\right)=\operatorname{tr}\left[\rho_{a b} \log \rho_{a b}\right]-\operatorname{tr}\left[\rho_{a b} \log \sigma_{a b}^{(c q)}\right] \\
= & S\left(\Pi_{a}\left(\rho_{a b}\right)\right)-S\left(\rho_{a b}\right)+S\left(\Pi_{a}\left(\rho_{a b}\right) \| \sigma_{a b}^{(c q)}\right) .
\end{aligned}
$$

We remark that a similar equation is established in block coherence theory [50].

\section{Bures distance}

The Bures distance is defined as $[42,43]$

$$
d_{B}(\rho, \sigma):=\sqrt{2-2 F(\rho, \sigma)},
$$

where $F(\rho, \sigma)$ is the fidelity $F(\rho, \sigma):=\operatorname{tr} \sqrt{\sqrt{\sigma} \rho \sqrt{\sigma}}$ between $\rho$ and $\sigma$. Since $F(\rho, \sigma) \in[0,1]$ and is unity iff $\rho=\sigma$, $d_{B}(\rho, \sigma)$ is nonnegative and vanishes iff $\rho=\sigma$. Moreover, the monotonicity and joint concavity of fidelity [3] implies that $d_{B}^{2}$ is contractive and jointly convex.

Unlike relative entropy, Bures distance is a bona fide distance on state space, that is, it is faithful, symmetric, and satisfies the triangle inequality. Moreover, it is Riemannian [51] and its metric is given in [51-53]. Quantum Fisher information-a quantum analog of Fisher information in statistics-is defined in terms of the Bures distance metric [54]. These properties of Bures distance enable it to quantify various quantum correlations including entanglement [36], discord [46, 55], coherence [11], etc.

\section{Entanglement}

An entanglement measure $E$ is a functional on $D(\mathcal{H})$ satisfying (1) Faithfulness: $E(\rho) \geq 0$, where the equality holds iff $\rho \in \mathcal{S}$, and (2) Monotonicity: $E(\rho) \geq E(\Phi(\rho))$ for 
any LOCC operation $\Phi$.

Bures distance of entanglement.-The Bures distance of entanglement is defined as the minimal square of Bures distance to separable states [36],

$$
E_{B}\left(\rho_{a b}\right):=\min _{\sigma_{a b} \in \mathcal{S}} d_{B}^{2}\left(\rho_{a b}, \sigma_{a b}\right) .
$$

Obviously, $E_{B}\left(\rho_{a b}\right)$ is nonnegative, and vanishes iff $\rho_{a b}$ is separable. Furthermore, $E_{B}$ is convex and non-increasing under LOCC operations [34, 37]. Note that for the bipartite pure state $|\psi\rangle=\sum_{i} \sqrt{\lambda_{i}}\left|x_{i}\right\rangle_{a}\left|y_{i}\right\rangle_{b}$ with $\lambda_{1} \geq \ldots \geq \lambda_{n}$, $E_{B}(|\psi\rangle)=2\left(1-\sqrt{\lambda_{1}}\right)$ [45]. In fact, if we assume that $\sigma_{a b} \in \mathcal{S}$ has a separable pure state decomposition $\sigma_{a b}=$ $\sum_{j} q_{j}\left|\phi_{j}\right\rangle_{a b}\left\langle\phi_{j}\right|$, then

$$
\begin{gathered}
F\left(|\psi\rangle, \sigma_{a b}\right)=\sqrt{\sum_{j} q_{j}\left|\left\langle\psi \mid \phi_{j}\right\rangle\right|^{2}} \\
\leq \sqrt{\sum_{j} q_{j}\left|\left\langle\psi \mid \phi_{\max }\right\rangle\right|^{2}}=\left|\left\langle\psi \mid \phi_{\max }\right\rangle\right|,
\end{gathered}
$$

where $\left|\left\langle\psi \mid \phi_{\max }\right\rangle\right|:=\max _{j}\left|\left\langle\psi \mid \phi_{j}\right\rangle\right|$ is the maximal over all $j$. Therefore, $E_{B}(|\psi\rangle)=2\left(1-\sqrt{\lambda_{1}}\right)$ and the corresponding closest separable state is $\left|x_{1}, y_{1}\right\rangle_{a b}$.

Convex roof of Bures distance of entanglement.-The convex roof of Bures distance of entanglement is defined as

$$
E_{B}^{c r}\left(\rho_{a b}\right):=\min _{p_{i},\left|\psi_{i}\right\rangle} \sum_{i} p_{i} E_{B}\left(\left|\psi_{i}\right\rangle\right),
$$

where the minimal is taken over all pure state decompositions $\rho_{a b}=\sum_{i} p_{i}\left|\psi_{i}\right\rangle\left\langle\psi_{i}\right|$.

\section{E. Quantum discord}

Asymmetric quantum discord.-A functional $\hat{D}$ on $D(\mathcal{H})$ is called a discord measure (asymmetric or local) if it satisfies the following properties:

(D1) $\hat{D}$ is faithful, i.e., $\hat{D}\left(\rho_{a b}\right) \geq 0$ and the equality holds if and only if $\rho_{a b} \in \mathcal{C Q}$.

(D2) $\hat{D}$ is non-increasing for any quantum operation on subsystem $b$, i.e., $\hat{D}\left(\rho_{a b}\right) \geq \hat{D}\left(I_{a} \otimes \Phi_{b}\left(\rho_{a b}\right)\right)$ for any local operation $\Phi_{b}$.

(D3) $\hat{D}$ is invariant under local unitary transformations, i.e., $\hat{D}\left(U_{a} \otimes U_{b} \rho_{a b} U_{a}^{\dagger} \otimes U_{b}^{\dagger}\right)=\hat{D}\left(\rho_{a b}\right)$ for any unitary $U_{a} \otimes U_{b}$ acting on $\mathcal{H}_{a} \otimes \mathcal{H}_{b}$.

(D4) $\hat{D}$ reduces to an entanglement monotone [56] for pure states.

Quantum discord has been studied extensively from different viewpoints in the forms of geometric discord, measurement-induced geometric discord [57] and geometric correlated partial coherence [49]. Quantum discord, obviously, captures quantum correlation beyond entanglement in the sense that a separable state may have nonzero quantum discord.

Symmetric quantum discord.-A functional $\tilde{D}$ in state space is called (symmetric or global) discord $[9,10]$ measure if it satisfies the following properties:

(D'1) $\tilde{D}$ is faithful, i.e., $\tilde{D}\left(\rho_{a b}\right) \geq 0$ and the equality holds if and only if $\rho_{a b} \in \mathcal{C C}$.

$\left(\mathrm{D}^{\prime} 2\right) \widetilde{D}$ is invariant under local unitary transformations, i.e., $\tilde{D}\left(U_{a} \otimes U_{b} \rho_{a b} U_{a}^{\dagger} \otimes U_{b}^{\dagger}\right)=\tilde{D}\left(\rho_{a b}\right)$ for any unitary acting on $\mathcal{H}_{a}$ and $\mathcal{H}_{b}$.

$\left(\mathrm{D}^{\prime} 3\right) \tilde{D}$ reduces to an entanglement monotone on pure states.

Bures distance of discord.-The Bures distance of discord is defined as the minimal square of Bures distance to classicalquantum states [46, 55],

$$
\hat{D}_{B}\left(\rho_{a b}\right):=\min _{\sigma_{a b} \in \mathcal{C} \mathcal{Q}} d_{B}^{2}\left(\rho_{a b}, \sigma_{a b}\right) .
$$

It has been shown that $\hat{D}_{B}$ is a discord measure, i.e., it satisfies the properties (D1-D4).

Remark 1. Quantum interferometric power (QIP) [58] is a measure of discord-type quantum correlation defined via quantum Fisher information, which is upper bounded by local quantum uncertainly (LQU) [59]. Actually, if subsystem " $a$ " is a qubit, LQU is equivalent to Hellinger distance of discord $[57,60]$, and is upper bounded by Bures distance of discord. As a result, QIP is also upper bounded by Bures distance of discord in $C^{2} \otimes C^{d}$ case.

\section{CHARACTERIZING ENTANGLEMENT VIA ASYMMETRIC QUANTUM DISCORD}

In this section, we propose the framework to quantify entanglement via quantum discord (asymmetric or local), and introduce several quantifiers with geometric discord and measurement-induced geometric discord.

\section{A. General results}

In this section, we explore and interpret the relationship between entanglement and quantum discord (asymmetric) over state extensions.

Definition 1. For a bipartite state $\rho_{a b} \in D(\mathcal{H})$, the minimal discord over state extensions is defined as

$$
\hat{\mathcal{E}}\left(\rho_{a b}\right):=\min _{\operatorname{tr}_{a^{\prime}}\left[\rho_{a a^{\prime} b}\right]=\rho_{a b}} \hat{D}\left(\rho_{a a^{\prime} b}\right),
$$

where the minimization is taken over all state extensions $\rho_{a a^{\prime} b}$ of $\rho_{a b}$ [41] along the bipartition $a a^{\prime}: b$ (see Fig. 1).

Remark 2. Squashed entanglement [40] and the conditional entanglement of mutual information [61], two additive entanglement measures, are defined using the notion of "over state extensions". Thus, characterizing quantum entanglement using extended systems has proved an important idea in entanglement theory. 


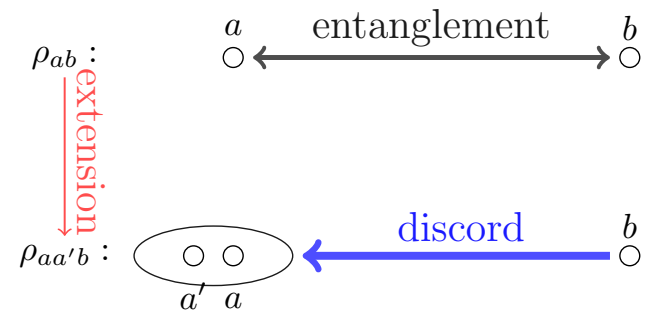

FIG. 1. The minimal discord of extended state $\rho_{a a^{\prime} b}$ between parties $a a^{\prime}$ and $b$ is a quantification of entanglement of $\rho_{a b}$ between $a$ and $b$.

Theorem 1. If $\hat{D}$ is a discord measure satisfying (D1-D4), the corresponding minimal discord, $\hat{\mathcal{E}}$, over state extensions has the following remarkable properties:

(E1) $\hat{\mathcal{E}}\left(\rho_{a b}\right) \geq 0$ with the equality if and only if $\rho \in \mathcal{S}$.

(E2) $\hat{\mathcal{E}}$ is invariant under local unitary transformations.

(E3) $\hat{\mathcal{E}}$ is nonincreasing under local partial trace,

$$
\hat{\mathcal{E}}\left(\rho_{a b}\right) \leq \hat{\mathcal{E}}\left(\rho_{a a_{1} b}\right)
$$

for any state extension $\rho_{a a_{1} b}$ of $\rho_{a b}$.

(E4) $\hat{\mathcal{E}}$ is nonincreasing under local operations in party a.

(E5) $\hat{\mathcal{E}}$ reduces to an entanglement monotone for pure states.

Proof. (E1) The nonnegativity of quantum discord implies that $\hat{\mathcal{E}}$ is always nonnegative. Moreover, any separable state $\rho_{a b}=\sum_{i} p_{i} \rho_{a}^{i} \otimes \rho_{b}^{i}$ can be embedded into a larger classicalquantum state $\rho_{a a^{\prime} b}=\sum_{i} p_{i}\left|\alpha_{i}\right\rangle_{a a^{\prime}}\left\langle\alpha_{i}\right| \otimes \rho_{b}^{i}$ such that $\rho_{a b}=t r_{a^{\prime}}\left[\rho_{a a^{\prime} b}\right]$, where $a^{\prime}$ is the ancillary system pertinent to party $a$ and $\left|\alpha_{i}\right\rangle_{a a^{\prime}}$ is the purification of $\rho_{a}^{i}$ for each $i$ [30]. However, an entangled state does not admit such an extension. Therefore, $\hat{\mathcal{E}}$ is faithful in separable states.

(E2) Assuming $\rho_{a a^{\prime} b}$ is the state extension of $\rho_{a b}, t r_{a^{\prime}}\left[U_{a} \otimes\right.$ $\left.U_{b} \rho_{a a^{\prime} b} U_{a}^{\dagger} \otimes U_{b}^{\dagger}\right]=U_{a} \otimes U_{b} \rho_{a b} U_{a}^{\dagger} \otimes U_{b}^{\dagger}$ and the local unitary invariance of $\hat{D}$ implies that $\hat{\mathcal{E}}\left(\rho_{a b}\right) \geq \hat{\mathcal{E}}\left(U_{a} \otimes U_{b} \rho_{a b} U_{a}^{\dagger} \otimes\right.$ $\left.U_{b}^{\dagger}\right)$. On the contrary, we can also show $\hat{\mathcal{E}}\left(\rho_{a b}\right) \leq \hat{\mathcal{E}}\left(U_{a} \otimes\right.$ $\left.U_{b} \rho_{a b} U_{a}^{\dagger} \otimes U_{b}^{\dagger}\right)$ implying that $\hat{\mathcal{E}}$ is invariant under local unitary transformation.

(E3) This follows trivially because any state extension $\rho_{a a_{1} a^{\prime} b}$ of state $\rho_{a a_{1} b}$ is also the extension of $\rho_{a b}$.

(E4) Using Stinespring representation [62], the local operation in party $a$ can be realized by adding a pure state ancilla, followed by a global unitary operation and tracing out the ancilla system, i.e., $\sum_{i} K_{i}^{a} \rho_{a b} K_{i}^{a \dagger}=t r_{a_{1}} U_{a a_{1}}\left(\rho_{a b} \otimes|0\rangle_{a_{1}}\langle 0|\right) U_{a a_{1}}^{\dagger}$. Therefore, one has

$$
\begin{aligned}
\hat{\mathcal{E}}\left(\rho_{a b}\right) & \geq \hat{\mathcal{E}}\left(\rho_{a b} \otimes|0\rangle_{a_{1}}\langle 0|\right) \\
& =\hat{\mathcal{E}}\left(U_{a a_{1}} \rho_{a b} \otimes|0\rangle_{a_{1}}\langle 0| U_{a a_{1}}^{\dagger}\right) \\
& \geq \hat{\mathcal{E}}\left(\sum_{i} K_{i}^{a} \rho_{a b} K_{i}^{a \dagger}\right)
\end{aligned}
$$

where the inequality in the first and the third lines follows from property (E3).
(E5) Firstly, combining the definition of $\hat{\mathcal{E}}$ and property (E3),

$$
\hat{\mathcal{E}}(|\psi\rangle) \geq \min _{|\phi\rangle} \hat{D}(|\psi\rangle \otimes|\phi\rangle) \geq \hat{D}(|\psi\rangle)=E(|\psi\rangle) .
$$

Further, considering the special case, that is, the extension space is one-dimensional, $\hat{\mathcal{E}}(|\psi\rangle)=\hat{D}(|\psi\rangle)=E(|\psi\rangle)$.

Remark 3. The above results provide an alternative avenue to understand quantum entanglement from the viewpoint of discord over state extensions. We can see that $\hat{\mathcal{E}}$ is a good candidate of an entanglement measure. Moreover, $\hat{D}$ reduces to $\hat{\mathcal{E}}$ for pure states.

We call a nonnegative bivariate function $d$ on state space pseudo-distance if $d(\rho, \sigma)=0$ iff $\rho=\sigma$, and call $d$ contractive, if it satisfies $d(\rho, \sigma) \geq d(\Phi(\rho), \Phi(\sigma))$ for any quantum operation $\Phi$ and $\rho, \sigma \in D(\mathcal{H})$. Relative entropy and Bures distance are examples of pseudo-distances. We will simply write "distance" for simplicity.

Definition 2. For a bipartite state $\rho_{a b} \in D(\mathcal{H})$, the minimal geometric discord over state extensions (GDSE) is defined as

$$
\hat{\mathcal{E}}_{d}\left(\rho_{a b}\right):=\min _{\sigma_{a a^{\prime} b} \in \mathcal{C} \mathcal{Q} \operatorname{tr}_{a^{\prime}}\left[\rho_{a a^{\prime} b}\right]=\rho_{a b}} d\left(\rho_{a a^{\prime} b}, \sigma_{a a^{\prime} b}\right),
$$

where the minimization is taken over all extended states $\rho_{a a^{\prime} b}$ and classical-quantum states in $D\left(\mathcal{H}_{a a^{\prime} b}\right)$. Here $d$ is a contractive distance in $D\left(\mathcal{H}_{a a^{\prime} b}\right)$. Furthermore, the minimal measurement-induced geometric discord over state extensions (MIDSE) is defined as

$$
\hat{\mathcal{E}}_{d}^{\prime}\left(\rho_{a b}\right):=\min _{\Pi_{a a^{\prime}} \operatorname{tr}_{a^{\prime}}\left[\rho_{a a^{\prime} b}\right]=\rho_{a b}} d\left(\rho_{a a^{\prime} b}, \Pi_{a a^{\prime}} \otimes I_{b}\left(\rho_{a a^{\prime} b}\right)\right)
$$

where the minimization is taken over extended states and local projection in subsystem $a a^{\prime}$.

Remark 4. For both relative entropy and Bures distance, the corresponding quantifiers of discord satisfy (D1-D4). Theorem 1 then implies that the corresponding GDSE and MIDSE are good candidates of entanglement measures.

Next, we consider the quantification of entanglement by performing partial trace; a slight modification of Eq. (11). Define quantum correlation by

$$
\check{\mathcal{E}}_{d}\left(\rho_{a b}\right):=\min _{\sigma_{a a^{\prime} b} \in \mathcal{C} \mathcal{Q}} d\left(\rho_{a b}, t r_{a^{\prime}} \sigma_{a a^{\prime} b}\right),
$$

where the minimum is taken over all classical-quantum state in $D\left(\mathcal{H}_{a a^{\prime} b}\right)$. Obviously, $\check{\mathcal{E}}_{d}$ is equivalent to $E_{d}:=$ $\min _{\sigma_{a b} \in \mathcal{S}} d\left(\rho_{a b}, \sigma_{a b}\right)$, which is the corresponding entanglement quantification of distance $d$. In other words, geometric entanglement can be linked to discord over state extensions. For measurement-induced geometric discord, however, it is not the trivial case, as shown below.

Definition 3. For $\rho_{a b} \in D\left(\mathcal{H}_{a b}\right)$, we define a quantity

$$
\check{\mathcal{E}}_{d}^{\prime}\left(\rho_{a b}\right):=\min _{\Pi_{a a^{\prime}} \operatorname{tr}_{a^{\prime}}\left[\rho_{a a^{\prime} b}\right]=\rho_{a b}} d\left(\rho_{a b}, t r_{a^{\prime}}\left[\Pi_{a a^{\prime}} \otimes I_{b}\left(\rho_{a a^{\prime} b}\right)\right]\right),
$$

where the minimal is taken over all extended states $\rho_{a a^{\prime} b}$ and local projection in subsystem $a a^{\prime}$. 
This quantity is related to the previous ones, via the following inequalities.

Theorem 2. For $\rho_{a b} \in D\left(\mathcal{H}_{a b}\right)$,

$$
\begin{aligned}
& \hat{\mathcal{E}}_{d}^{\prime}\left(\rho_{a b}\right) \geq \hat{\mathcal{E}}_{d}\left(\rho_{a b}\right) \geq E_{d}\left(\rho_{a b}\right), \\
& \check{\mathcal{E}}_{d}^{\prime}\left(\rho_{a b}\right) \geq \check{\mathcal{E}}_{d}\left(\rho_{a b}\right)=E_{d}\left(\rho_{a b}\right),
\end{aligned}
$$

and

$$
\begin{aligned}
& \hat{\mathcal{E}}_{d}^{\prime}\left(\rho_{a b}\right) \geq \check{\mathcal{E}}_{d}^{\prime a}\left(\rho_{a b}\right), \\
& \hat{\mathcal{E}}_{d}\left(\rho_{a b}\right) \geq \check{\mathcal{E}}_{d}\left(\rho_{a b}\right)=E_{d}\left(\rho_{a b}\right) .
\end{aligned}
$$

Proof. These inequalities can be derived from the definition directly.

\section{B. Characterization with Bures distance discord}

Here, we prove two important theorems related to the minimal Bures distance discord, $\hat{\mathcal{E}}_{B}$, over state extensions. In the following theorem we show that $\mathcal{E}_{B}^{a}$ is convex.

Theorem 3. $\hat{\mathcal{E}}_{B}$ is convex,

$$
\hat{\mathcal{E}}_{B}\left(\sum_{i} p_{i} \rho_{a b}^{i}\right) \leq \sum_{i} p_{i} \hat{\mathcal{E}}_{B}\left(\rho_{a b}^{i}\right),
$$

where $p_{i}$ are probabilities and $\rho_{a b}^{i}$ are bipartite states shared between parties $a$ and $b$.

Proof. See Appendix VI A.

Using Theorem 3, we arrive at another theorem below.

Theorem 4. For $\rho_{a b} \in D(\mathcal{H})$, the minimal Bures distance of discord over state extensions is equivalent to the Bures distance of entanglement,

$$
\hat{\mathcal{E}}_{B}\left(\rho_{a b}\right)=E_{B}\left(\rho_{a b}\right) .
$$

Proof. Since $E_{B}\left(\rho_{a b}\right)=E_{B}^{c r}\left(\rho_{a b}\right)$ [45], we just need to show that for each $\rho_{a b} \in D(\mathcal{H})$, we have

$$
E_{B}\left(\rho_{a b}\right) \leq \hat{\mathcal{E}}_{B}\left(\rho_{a b}\right) \leq E_{B}^{c r}\left(\rho_{a b}\right) .
$$

See Appendix VI B for the complete proof.

Having established these results, Theorem 2 for the Bures distance together with Theorem 4 imply

$$
\hat{\mathcal{E}}_{B}^{\prime}\left(\rho_{a b}\right) \geq \check{\mathcal{E}}_{B}^{\prime}\left(\rho_{a b}\right) \geq E_{B}\left(\rho_{a b}\right)=\hat{\mathcal{E}}_{B}\left(\rho_{a b}\right)=\check{\mathcal{E}}_{B}\left(\rho_{a b}\right) .
$$

\section{Operational meaning of Bures distance of entanglement}

The quest for an operational meaning or interpretation of an entanglement measure lies at the very heart of the entanglement theory. While entanglement of formation, distillable entanglement or entanglement cost are measures of entanglement having an operational meaning $[1,3]$, the Bures distance of entanglement is an entanglement measure defined from the geometric viewpoint and its physical meaning is not clear. By means of the equivalence in Theorem 4 , and the operational meaning of Bures distance of discord [46], we provide an operational meaning of the Bures distance of entanglement.

Let us briefly review the ambiguous quantum state discrimination (QSD) protocol [63]. Suppose Alice chooses a state $\rho_{i}$ from a set of states $\left\{\rho_{i}\right\}$ with probability $\eta_{i}$ and sends it to Bob, who determines which state he receives by performing a positive-perator-valued measure (POVM). Since the probability to get the result $j$ with given state $\rho_{i}$ is $p(j \mid i)=\operatorname{tr}\left(M_{j} \rho_{i}\right)$, then the corresponding optimal success probability is

$$
P_{s}^{o p t}\left(\left\{\rho_{i}, \eta_{i}\right\}_{i=1}^{N}\right):=\max _{M_{i}} \sum_{i} \eta_{i} \operatorname{tr}\left(M_{i} \rho_{i}\right),
$$

where the maximization is done over all POVMs. Similarly, $P_{s}^{o p t}(v N)\left(\left\{\rho_{i}, \eta_{i}\right\}_{i=1}^{N}\right)=\max _{\Pi_{i}} \sum_{i} \eta_{i} t r\left(\Pi_{i} \rho_{i}\right)$ is the optimal success probability to discriminate $\left\{\rho_{i}, \eta_{i}\right\}_{i=1}^{N}$ with von Neumann measurement.

Based on the operational meaning of the Bures distance of asymmetric discord [46], the connection between geometric entanglement and QSD is as follows.

Corollary. For a bipartite quantum state $\rho_{a b} \in D(\mathcal{H})$, the square of the maximal fidelity to the set of separable states, $F\left(\rho_{a b}, \mathcal{S}\right):=\max _{\sigma_{a b} \in \mathcal{S}} F\left(\rho_{a b}, \sigma_{a b}\right)$, is equal to the optimal success probability to discriminate a set of quantum states with von Neumann measurement, i.e.,

$$
F^{2}\left(\rho_{a b}, \mathcal{S}\right)=\max _{\rho_{a a^{\prime} b},\left|\alpha_{i}\right\rangle} P_{s}^{o p t(v N)}\left(\left\{\rho_{i}, \eta_{i}\right\}\right),
$$

where $\eta_{i}=\operatorname{tr}\left\langle\alpha_{i}\left|\rho_{a a^{\prime} b}\right| \alpha_{i}\right\rangle, \quad \rho_{i}=\eta_{i}^{-1} \sqrt{\rho_{a a^{\prime} b}}\left|\alpha_{i}\right\rangle\left\langle\alpha_{i}\right|$ $\sqrt{\rho_{a a^{\prime} b}}$ and the maximum is taken over all possible extended states $\rho_{a a^{\prime} b}$, von Neumann measurement $\Pi_{i}$ and orthogonal basis $\left\{\left|\alpha_{i}\right\rangle\right\}$ on $\mathcal{H}_{a a^{\prime}}$.

This offers an operational meaning to the Bures distance of entanglement.

\section{Characterization with relative entropy discord}

We define relative entropy of discord by $\hat{D}_{r}(\rho)$ := $\min _{\sigma \in \mathcal{C} \mathcal{Q}} S(\rho \| \sigma)$ and measurement-induced relative entropy of discord by $\hat{D}_{r}^{\prime}(\rho):=\min _{\Pi_{a}} S\left(\rho \| \Pi_{a}(\rho)\right)$.

Remark 5. In Ref. [11] the authors provided a framework to quantify quantum coherence with respect to a chosen orthogonal basis. Although discord and coherence seem different, they have essential similarities. Discord can be regarded as the basis-independent coherence [64], and relative entropy of 
discord is equivalent to the minimal relative entropy of partial coherence [65]. Moreover, quantum discord can also be explained as an upper bound to the quantum correlations generated from partial coherence via partial incoherent operations [66].

In the following theorem, we show that $\hat{D}_{r}$ and $\hat{D}_{r}^{\prime}$ are equivalent.

Theorem 5. For a bipartite state $\rho_{a b} \in D(\mathcal{H})$, the relative entropy of discord is equivalent to the measurement-induced relative entropy of discord, i.e.,

$$
\hat{D}_{r}\left(\rho_{a b}\right)=\hat{D}_{r}^{\prime}\left(\rho_{a b}\right)
$$

Proof. Since any classical-quantum state has the form $\sigma_{a b}^{(c q)}=$ $\sum_{i} p_{i}|i\rangle_{a}\langle i| \otimes \sigma_{b}^{i}$, using Eq. (2) we have

$$
\begin{aligned}
\hat{D}_{r}\left(\rho_{a b}\right) & =\min _{\pi_{a}^{i}} \min _{p_{i}, \sigma_{b}^{i}} S\left(\rho_{a b} \| \sigma_{a b}^{(c q)}\right) \\
& =\min _{\pi_{a}^{i}}\left[S\left(\Pi_{a}\left(\rho_{a b}\right)\right)-S\left(\rho_{a b}\right)+\min _{p_{i}, \sigma_{b}^{i}} S\left(\Pi_{a}\left(\rho_{a b}\right) \| \sigma_{a b}^{(c q)}\right)\right] \\
& =\min _{\pi_{a}^{i}} S\left(\Pi_{a}\left(\rho_{a b}\right)\right)-S\left(\rho_{a b}\right) \\
& =\min _{\pi_{a}^{i}} S\left(\rho_{a b} \| \Pi_{a}\left(\rho_{a b}\right)\right)=\hat{D}_{r}^{\prime}\left(\rho_{a b}\right)
\end{aligned}
$$

where $\Pi_{a}\left(\rho_{a b}\right)=\sum_{i}\left(|i\rangle_{a}\langle i| \otimes I_{b}\right) \rho_{a b}\left(|i\rangle_{a}\langle i| \otimes I_{b}\right)$.

Next, for the relative entropic quantities $E_{r}, \hat{\mathcal{E}}_{r}^{\prime}, \hat{\mathcal{E}}_{r}, \check{\mathcal{E}}_{r}^{\prime}$ and $\check{\mathcal{E}}_{r}$, where symbols have their usual meanings, Theorem 2 and Theorem 5 lead to the following result.

Theorem 6. For a bipartite state $\rho_{a b} \in D(\mathcal{H})$,

$$
\hat{\mathcal{E}}_{r}^{\prime}\left(\rho_{a b}\right)=\hat{\mathcal{E}}_{r}\left(\rho_{a b}\right) \geq E_{r}\left(\rho_{a b}\right)=\check{\mathcal{E}}_{r}^{\prime}\left(\rho_{a b}\right)=\check{\mathcal{E}}_{r}\left(\rho_{a b}\right) .
$$

Proof. From Theorem 5, we have

$$
\hat{\mathcal{E}}_{r}^{\prime}\left(\rho_{a b}\right)=\hat{\mathcal{E}}_{r}\left(\rho_{a b}\right)
$$

and

$$
\check{\mathcal{E}}_{r}^{\prime}\left(\rho_{a b}\right)=\check{\mathcal{E}}_{r}\left(\rho_{a b}\right)=E_{r}\left(\rho_{a b}\right) .
$$

The inequality follows from Theorem 2.

Remark 6. In Ref. [30], authors proved that $\check{\mathcal{E}}_{r}^{\prime}\left(\rho_{a b}\right)=$ $\min _{t r_{a}\left[\sigma_{a b}^{(s e p)}\right]=\rho_{b}} S\left(\rho_{a b} \| \sigma_{a b}^{(s e p)}\right) \geq E_{r}\left(\rho_{a b}\right)$, where the minimum is taken over all separable states $\sigma_{a b}^{(s e p)}$ with $\operatorname{tr}_{a}\left[\sigma_{a b}^{(s e p)}\right]=\rho_{b}$. Our results affirm that they are equal.

\section{CHARACTERIZING ENTANGLEMENT VIA SYMMETRIC DISCORD}

In this section, we quantify entanglement via symmetric or global quantum discord. In the symmetric or global quantum discord of a bipartite system, the local measurement is performed on both subsystems. Without further ado, all the contents developed in the asymmetric case will be recalled here, with the only difference that now quantum measurement or other treatment will be on both parties.

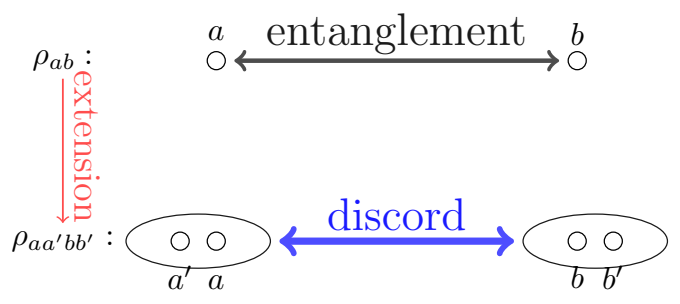

FIG. 2. The minimal discord of an extended state $\rho_{a a^{\prime} b b^{\prime}}$ shared between parties $a a^{\prime}$ and $b b^{\prime}$ is a quantification of entanglement of $\rho_{a b}$ between $a$ and $b$.

\section{A. General results}

Following, we define the minimal quantum discord over ]state extensions. In the asymmetric case, the state extension was in party $a$. Here we consider state extension in both parties.

Definition 4. For bipartite state $\rho_{a b} \in D(\mathcal{H})$, the minimal discord over state extensions is defined as

$$
\tilde{\mathcal{E}}\left(\rho_{a b}\right):=\min _{\operatorname{tr}_{a^{\prime} b^{\prime}}\left[\rho_{a a^{\prime} b b^{\prime}}\right]=\rho_{a b}} \tilde{D}\left(\rho_{a a^{\prime} b b^{\prime}}\right),
$$

where the minimization is taken along the bipartition $a a^{\prime}: b b^{\prime}$ with $\operatorname{tr}_{a^{\prime}} \rho_{a a^{\prime} b b^{\prime}}=\rho_{a b}$ (see Fig. 2).

Theorem 7. If $\tilde{D}$ is a symmetric discord measure satisfying $\left(D^{\prime} 1-D^{\prime} 3\right)$, the minimal discord, $\tilde{\mathcal{E}}$, over state extensions has the following desirable and remarkable properties:

(E' 1$) \tilde{\mathcal{E}}\left(\rho_{a b}\right) \geq 0$ with the equality if and only if $\rho \in \mathcal{C C}$.

$\left(E^{\prime} 2\right) \tilde{\mathcal{E}}$ is invariant under local unitary transformations.

$\left(E^{\prime} 3\right) \tilde{\mathcal{E}}$ is nonincreasing under local partial trace,

$$
\tilde{\mathcal{E}}\left(\rho_{a b}\right) \leq \tilde{\mathcal{E}}\left(\rho_{a a_{1} b b_{1}}\right),
$$

for any state extension $\rho_{a a_{1} b b_{1}}$ of $\rho_{a b}$.

$\left(E^{\prime} 4\right) \tilde{\mathcal{E}}$ is nonincreasing under local operations.

$\left(E^{\prime} 5\right) \tilde{\mathcal{E}}$ reduces to an entanglement monotone for pure states.

Proof. The proof is similar to that of Theorem 1.

\section{B. Minimal Bures distance of symmetric discord}

In this part, we define the minimal Bures distance of symmetric discord.

Definition 5. For a bipartite state $\rho_{a b} \in \mathcal{H}$, the minimal Bures distance of discord over state extensions is defined as

$$
\tilde{\mathcal{E}}_{B}\left(\rho_{a b}\right):=\min _{\sigma \in \mathcal{C} \mathcal{C} \operatorname{tr}_{a^{\prime} b^{\prime}}\left[\rho_{a a^{\prime} b b^{\prime}}\right]=\rho_{a b}} d_{B}^{2}\left(\rho_{a a^{\prime} b b^{\prime}}, \sigma\right),
$$

where the minimum is taken with respect to the bipartition $a a^{\prime}: b b^{\prime}$. 
Following, we state that the minimal Bures distance of symmetric discord is convex, and is equivalent to the Bures distance of entanglement.

Theorem 8. $\tilde{\mathcal{E}}_{B}$ is convex,

$$
\tilde{\mathcal{E}}_{B}\left(\sum_{i} p_{i} \rho_{a b}^{i}\right) \leq \sum_{i} p_{i} \tilde{\mathcal{E}}_{B}\left(\rho_{a b}^{i}\right),
$$

where $p_{i}$ are probabilities and $\rho_{a b}^{i}$ are bipartite states shared between parties $a$ and $b$.

The proof is similar to that of Theorem 3. With above Theorem, one has the following result.

Theorem 9. For $\rho_{a b} \in D(\mathcal{H}), \tilde{\mathcal{E}}_{B}\left(\rho_{a b}\right)=E_{B}\left(\rho_{a b}\right)$.

The proof is similar to that of Theorem 4.

Remark 7. It is interesting to note that $E_{B}\left(\rho_{a b}\right)=\hat{\mathcal{E}}_{B}\left(\rho_{a b}\right)=$ $\tilde{\mathcal{E}}_{B}\left(\rho_{a b}\right)$, which means that Bures distance of entanglement is equivalent to the minimal Bures distance of discord over state extensions, both on one subsystem and two subsystems.

Remark 8. It was Luo who first proposed to quantify entanglement as the minimal quantum discord over state extensions [31]. Above theorem offers an affirmative evidence that this kind of entanglement quantification is consistent with the previous entanglement measures. However, it is not clear whether this equivalence still holds for other entanglement measures such as entanglement measures based on distances [1] and relative entropy of entanglement [36].

\section{More definitions}

Definition 6. For a bipartite state $\rho_{a b} \in D(\mathcal{H})$, the minimal geometric discord over state extensions (GDSE) is defined as

$$
\tilde{\mathcal{E}}_{d}\left(\rho_{a b}\right):=\min _{\sigma_{a a^{\prime} b b^{\prime}} \in \mathcal{C C} \operatorname{cr}_{a^{\prime} b^{\prime}}\left[\rho_{a a^{\prime} b b^{\prime}}\right]=\rho_{a b}} d\left(\rho_{a a^{\prime} b b^{\prime}}, \sigma_{a a^{\prime} b b^{\prime}}\right),
$$

where the minimum is taken with respect to the bipartition $a a^{\prime}: b b^{\prime}$. Moreover, the minimal measurement-induced geometric discord over state extensions (MIDSE) is defined as

$$
\tilde{\mathcal{E}}_{d}^{\prime}\left(\rho_{a b}\right):=\min _{\Pi_{a a^{\prime}}, \Pi_{b b^{\prime}}} \min _{\mathrm{tr}_{a^{\prime} b^{\prime}}\left[\rho_{a a^{\prime} b b^{\prime}}\right]=\rho_{a b}} d\left(\rho_{a a^{\prime} b b^{\prime}}, \Pi_{a a^{\prime}} \otimes \Pi_{b b^{\prime}}\left(\rho_{a a^{\prime} b b^{\prime}}\right)\right),
$$

where the minimum is taken over all local projections in both subsystems $a a^{\prime} \& b b^{\prime}$ and along the bipartition $a a^{\prime}: b b^{\prime}$.

Remark 9. For relative entropy or Bures distance, the corresponding geometric discord satisfies $\left(\mathrm{D}^{\prime} 1-D^{\prime} 3\right)$. From Theorem 7 , the corresponding quantification is a good candidate of entanglement measure.

Next, let us consider the quantification by performing partial trace,

$$
\breve{\mathcal{E}}_{d}\left(\rho_{a b}\right):=\min _{\sigma_{a a^{\prime} b b^{\prime}} \in \mathcal{C C}} d\left(\rho_{a b}, t r_{a^{\prime} b^{\prime}} \sigma_{a a^{\prime} b b^{\prime}}\right)
$$

where the minimum is taken over all classical-classical states. It is easy to see that $\breve{\mathcal{E}}_{d}$ is equivalent to the general geometric entanglement measure $E_{d}$, which is defined as the minimal distance between the state and the set of separable states. Nevertheless, it is not the case for the measurement-induced geometric discord, as shown below.

Definition 7. For $\rho_{a b} \in D\left(\mathcal{H}_{a b}\right)$, we define a quantity $\breve{\mathcal{E}}_{d}^{\prime}\left(\rho_{a b}\right):=\min _{\Pi_{a a^{\prime}}, \Pi_{b b^{\prime}}} \min _{\mathrm{tr}_{a^{\prime} b^{\prime}}\left[\rho_{a a^{\prime} b b^{\prime}}\right]=\rho_{a b}} d\left(\rho_{a b}, \operatorname{tr}_{a^{\prime} b^{\prime}}\right.$ $\left.\left[\Pi_{a a^{\prime}} \otimes \Pi_{b b^{\prime}}\left(\rho_{a a^{\prime} b b^{\prime}}\right)\right]\right)$, where the minimum is taken over all extended states $\rho_{a a^{\prime} b b^{\prime}}$ and local projection in both subsystems $a a^{\prime}$ and $b b^{\prime}$.

Certainly, we obtain $\breve{\mathcal{E}}_{d}^{\prime}\left(\rho_{a b}\right) \geq E_{d}\left(\rho_{a b}\right)$ for any $\rho_{a b} \in$ $D\left(\mathcal{H}_{a b}\right)$. Moreover, based on the discussion in Remark 6, the equality holds for relative entropy. And whether the equality holds for other distances as well would be an interesting investigation.

In conclusion, we have the following result.

Theorem 10. For $\rho_{a b} \in D\left(\mathcal{H}_{a b}\right)$, the following inequalities are true.

$$
\begin{aligned}
& \tilde{\mathcal{E}}_{d}^{\prime}\left(\rho_{a b}\right) \geq \tilde{\mathcal{E}}_{d}\left(\rho_{a b}\right) \geq E_{d}\left(\rho_{a b}\right), \\
& \breve{\mathcal{E}}_{d}^{\prime}\left(\rho_{a b}\right) \geq \breve{\mathcal{E}}_{d}\left(\rho_{a b}\right)=E_{d}\left(\rho_{a b}\right),
\end{aligned}
$$

and

$$
\begin{aligned}
& \tilde{\mathcal{E}}_{d}^{\prime}\left(\rho_{a b}\right) \geq \breve{\mathcal{E}}_{d}^{\prime}\left(\rho_{a b}\right), \\
& \tilde{\mathcal{E}}_{d}\left(\rho_{a b}\right) \geq \breve{\mathcal{E}}_{d}\left(\rho_{a b}\right)=E_{d}\left(\rho_{a b}\right) .
\end{aligned}
$$

Proof. The inequalities can be derived directly from the definitions.

\section{Characterization with Bures distance discord and relative entropy discord}

For the Bures distance, Theorem 9 implies

$$
\tilde{\mathcal{E}}_{B}^{\prime}\left(\rho_{a b}\right) \geq \breve{\mathcal{E}}_{B}^{\prime}\left(\rho_{a b}\right) \geq E_{B}\left(\rho_{a b}\right)=\mathcal{E}_{B}\left(\rho_{a b}\right)=\breve{\mathcal{E}}_{B}\left(\rho_{a b}\right) .
$$

and for relative entropy,

$$
\tilde{\mathcal{E}}_{r}^{\prime}\left(\rho_{a b}\right)=\tilde{\mathcal{E}}_{r}\left(\rho_{a b}\right) \geq E_{r}\left(\rho_{a b}\right)=\breve{\mathcal{E}}_{r}^{\prime}\left(\rho_{a b}\right)=\breve{\mathcal{E}}_{r}^{\prime a}\left(\rho_{a b}\right)
$$

\section{CONCLUSION}

In this paper, we have proposed a framework to quantify entanglement from the viewpoint of discord, which unifies previous works including [29-31]. Several quantifications of entanglement are introduced based on quantum discord over state extensions.

Especially, for the Bures distance, we prove that the minimal discord (both asymmetric and symmetric) over state extensions is equivalent to the Bures distance of entanglement, which not only establishes an equivalence between this 
kind of entanglement quantification with existing entanglement measure, and but also provides an operational meaning for the Bures distance of entanglement.

Moreover, for relative entropy, by proving that the corresponding discord measure is equivalent to the measurement induced relative entropy of discord, we show that the MIDSE (both asymmetric and symmetric) is equivalent to the relative entropy of entanglement, which reinforces the result in Ref. [30].

\section{ACKNOWLEDGMENTS}

This project is supported in part by the Postdoctoral Science Foundation of China (2021M702864), the National Natural Science Foundation of China (Grants No. 61876195, No. 61572532, No. 12050410232, No. 12031004, and No. 61877054), the Natural Science Foundation of Guangdong Province of China (Grant No. 2017B030311011), Jiangxi Provincial Natural Science Foundation (Grant No. 20202BAB201001) and the Fundamental Research Foundation for the Central Universities (Project No.K20210337).
[1] R. Horodecki, P. Horodecki, M. Horodecki, and K. Horodecki, Quantum entanglement, Rev. Mod. Phys. 81, 865 (2009).

[2] K. Modi, A. Brodutch, H. Cable, T. Paterek, and V. Vedral, The classical-quantum boundary for correlations: Discord and related measures, Rev. Mod. Phys. 84, 1655 (2012).

[3] M. A. Nielsen and I. L. Chuang, Quantum Computation and Quantum Information (Cambridge University Press, 2010).

[4] S. D. Bartlett, T. Rudolph, and R. W. Spekkens, Reference frames, superselection rules, and quantum information, Rev. Mod. Phys. 79, 555 (2007).

[5] E. Chitambar and G. Gour, Quantum resource theories, Rev. Mod. Phys. 91, 025001 (2019).

[6] A. Streltsov, G. Adesso, and M. B. Plenio, Colloquium: Quantum coherence as a resource, Rev. Mod. Phys. 89, 041003 (2017).

[7] H. Ollivier and W. H. Zurek, Quantum Discord: A Measure of the Quantumness of Correlations, Phys. Rev. Lett. 88, 017901 (2001).

[8] L. Henderson and V. Vedral, Classical, quantum and total correlations, J. Phys. A: Math. and Gen. 34, 6899 (2001).

[9] C. C. Rulli and M. S. Sarandy, Global quantum discord in multipartite systems, Phys. Rev. A. 84, 042109 (2011).

[10] J. Xu, Analytical expressions of global quantum discord for two classes of multi-qubit states, Phys. Lett. A 377, 238-242 (2013).

[11] T. Baumgratz, M. Cramer, and M. B. Plenio, Quantifying Coherence, Phys. Rev. Lett. 113, 140401 (2014).

[12] A. Streltsov, S. Rana, P. Boes, and J. Eisert, Structure of the Resource Theory of Quantum Coherence, Phys. Rev. Lett. 119, 140402 (2017).

[13] G. Saxena, E. Chitambar, and G. Gour, Dynamical resource theory of quantum coherence, Phys. Rev. Research 2, 023298 (2020).

[14] A. Datta, S. T. Flammia, and C. M. Caves, Entanglement and the power of one qubit, Phys. Rev. A 72, 042316 (2005).

[15] A. Datta, A. Shaji, and C. M. Caves, Quantum Discord and the Power of One Qubit, Phys. Rev. Lett. 100, 050502 (2008).

[16] T. S. Cubitt, F. Verstraete, W. Dür, and J. I. Cirac, Separable States Can Be Used To Distribute Entanglement, Phys. Rev. Lett. 91, 037902 (2003).

[17] M. Koashi and A. Winter, Monogamy of quantum entanglement and other correlations, Phys. Rev. A 69, 022309 (2004).

[18] M. Cornelio, M. Oliveira and F. Fanchini, Entanglement Irreversibility from Quantum Discord and Quantum Deficit, Phys. Rev. Lett.107, 020502 (2011).

[19] F. Fanchini, M. Cornelio, M. Oliveira and A. Caldeira, Conservation law for distributed entanglement of formation and quantum discord, Phys. Rev. A 84, 012313 (2011).
[20] L.-X. Cen, X.-Q. Li, J. Shao, and Y. Yan, Quantifying quantum discord and entanglement of formation via unified purifications, Phys. Rev. A 83, 054101 (2011).

[21] G. Adesso and A. Datta, Quantum versus Classical Correlations in Gaussian States, Phys. Rev. Lett. 105, 030501 (2010).

[22] D. Cavalcanti, L. Aolita, S. Boixo, K. Modi, M. Piani, and A. Winter, Operational interpretations of quantum discord, Phys. Rev. A 83, 032324 (2011).

[23] M. Piani, S. Gharibian, G. Adesso, J. Calsamiglia, P. Horodecki, and A. Winter, All Nonclassical Correlations Can Be Activated into Distillable Entanglement, Phys. Rev. Lett. 106, 220403 (2011).

[24] V. Madhok and A. Datta, Interpreting quantum discord through quantum state merging, Phys. Rev. A 83, 032323 (2011).

[25] A. Streltsov, H. Kampermann, and D. Bruß, Linking Quantum Discord to Entanglement in a Measurement, Phys. Rev. Lett. 106, 160401 (2011).

[26] A. Streltsov, H. Kampermann, and D. Bruß, Quantum Cost for Sending Entanglement, Phys. Rev. Lett. 108, 250501 (2012).

[27] M. Piani and G. Adesso, Quantumness of correlations revealed in local measurements exceeds entanglement, Phys. Rev. A 85, 040301 (2012).

[28] T. K. Chuan, J. Maillard, K. Modi, T. Paterek, M. Paternostro, and M. Piani, Quantum Discord Bounds the Amount of Distributed Entanglement, Phys. Rev. Lett. 109, 070501 (2012).

[29] N. Li and S. Luo, Classical states versus separable states, Phys. Rev. A 78, 024303 (2008).

[30] A. R. U. Devi and A. K. Rajagopal, Generalized Information Theoretic Measure to Discern the Quantumness of Correlations, Phys. Rev. Lett. 100, 140502 (2008).

[31] S. Luo, Entanglement as minimal discord over state extensions, Phys. Rev. A 94, 032129 (2016).

[32] K. C. Tan, H. Kwon, C.-Y. Park, and H. Jeong, Unified view of quantum correlations and quantum coherence, Phys. Rev. A 94, 022329 (2016).

[33] K. C. Tan and H. Jeong, Entanglement as the Symmetric Portion of Correlated Coherence, Phys. Rev. Lett. 121, 220401 (2018).

[34] C. H. Bennett, D. P. DiVincenzo, J. A. Smolin, and W. K. Wootters, Mixed-state entanglement and quantum error correction, Phys. Rev. A 54, 3824 (1996).

[35] C. H. Bennett, G. Brassard, S. Popescu, B. Schumacher, J. A. Smolin, and W. K. Wootters, Purification of Noisy Entanglement and Faithful Teleportation via Noisy Channels, Phys. Rev. Lett. 78, 2031 (1997).

[36] V. Vedral, M. B. Plenio, M. A. Rippin, and P. L. Knight, Quantifying Entanglement, Phys. Rev. Lett. 78, 2275 (1997).

[37] V. Vedral and M. B. Plenio, Entanglement measures and purifi- 
cation procedures, Phys. Rev. A 57, 1619 (1998).

[38] G. Vidal and R. Tarrach, Robustness of entanglement, Phys. Rev. A 59, 141 (1999).

[39] R. R. Tucci, Entanglement of distillation and conditional mutual information, arXiv:quant-ph/0202144 [quant-ph].

[40] M. Christandl and A. Winter, Squashed Entanglement: An Additive Entanglement Measure, J. Math. Phys. 45, 829 (2004).

[41] We call $\rho_{a a^{\prime} b}$ an extension of quantum state $\rho_{a b}$ if it satisfies $\operatorname{tr}_{a^{\prime}}\left[\rho_{a a^{\prime} b}\right]=\rho_{a b}$. Obviously, the purification of a quantum state is an example of state extension. By "over state extensions" we mean that optimization runs over all extended states $\rho_{a a^{\prime} b}$ of $\rho_{a b}$.

[42] D. Bures, An Extension of Kakutani's Theorem on Infinite Product Measures to the Tensor Product of Semifinite $w^{*}$-Algebras, Trans. Amer. Math. Soc. 135, 199 (1969).

[43] A. Uhlmann, The "transition probability" in the state space of $a *$-algebra, Rep. Math. Phys. 9, 273 (1976).

[44] T.-C. Wei and P. M. Goldbart, Geometric measure of entanglement and applications to bipartite and multipartite quantum states, Phys. Rev. A 68, 042307 (2003).

[45] A. Streltsov, H. Kampermann, and D. Bruß, Linking a distance measure of entanglement to its convex roof, New J. Phys. 12, 123004 (2010).

[46] D. Spehner and M. Orszag, Geometric quantum discord with Bures distance, New J. Phys. 15, 103001 (2013).

[47] B. Dakić, V. Vedral, and C. Brukner, Necessary and Sufficient Condition for Nonzero Quantum Discord, Phys. Rev. Lett. 105, 190502 (2010).

[48] S. Luo, Using measurement-induced disturbance to characterize correlations as classical or quantum, Phys. Rev. A 77, 022301 (2008).

[49] C. Xiong, S. Kim, and D. Qiu, Characterizing quantum correlation with the correlated part of partial coherence, Phys. Rev. A 100, 032334 (2019).

[50] J. Aberg, Quantifying Superposition, arXiv:quantph/0612146v1.

[51] D. Spehner, Quantum correlations and distinguishability of quantum states, J. Math. Phys. 55, 075211 (2014).

[52] M. Hübner, Explicit computation of the Bures distance for density matrices, Phys. Lett. A 163, 0375-9601 (1992).

[53] H.-J. Sommers and K. Zyczkowski, Bures volume of the set of mixed quantum states, J. Phys. A: Math. Gen. 36, 10083 (2003).

[54] S. Braunstein and C. Caves, Statistical distance and the geometry of quantum states, Phys. Rev. Lett. 72, 3439 (1994).

[55] B. Aaronson, R. Lo Franco, and G. Adesso, Comparative investigation of the freezing phenomena for quantum correlations under nondissipative decoherence, Phys. Rev. A 88, 012120 (2013).

[56] G. Vidal, Entanglement monotones, J. Mod. Opt 47, 355-376 (2013).

[57] W. Roga, D. Spehner, and F. Illuminati, Geometric measures of quantum correlations: characterization, quantification, and comparison by distances and operations, J. Phys. A: Math. Theor. 49, 235301 (2016).

[58] D. Girolami, A. Souza, V. Giovannetti, et al., Quantum Discord Determines the Interferometric Power of Quantum States, Phys. Rev. Lett 112, 210401 (2014)

[59] D. Girolami, T. Tufarelli and G. Adesso, Characterizing Nonclassical Correlations via Local Quantum Uncertainty, Phys. Rev. Lett 110, 240402 (2013)

[60] L. Chang and S. Luo, Remedying the local ancilla problem with geometric discord, Phys. Rev. A 87, 062303 (2013).

[61] D. Yang, M. Horodecki, and Z. Wang, An Additive and Operational Entanglement Measure: Conditional Entanglement of
Mutual Information, Phys. Rev. Lett 101, 140501 (2008)

[62] W. F. Stinespring, Positive Functions on $C^{*}$-Algebras, Proc. Amer. Math. Soc. 6, 211 (1955).

[63] J. A. Bergou, U. Herzog, and M. Hillery, 11 Discrimination of Quantum States, in Quantum State Estimation, edited by M. Paris and J. Řeháček (Springer, Berlin, Heidelberg, 2004) pp. 417-465.

[64] Y. Yao, X. Xiao, L. Ge, and C. Sun, Quantum coherence in multipartite systems, Phys. Rev. A 92, 022112 (2015).

[65] Y. Sun, Y. Mao and S. Luo, From quantum coherence to quantum correlations, Euro. Phys. Lett 118, 60007 (2017).

[66] S. Kim, L. Li, A. Kumar, and Junde Wu, Interrelation between partial coherence and quantum correlations, Phys. Rev. A 98, 022306 (2018).

\section{APPENDIX}

\section{A. Proof of Theorem 3}

Proof. Note that

$$
\rho_{a a^{\prime} a^{\prime \prime} b}:=\sum_{i} p_{i} \rho_{a a^{\prime} b}^{i} \otimes|i\rangle_{a^{\prime \prime}}\langle i|
$$

is a state extension of $\rho_{a b}=\sum_{i} p_{i} \rho_{a b}^{i}$ whenever $\rho_{a a^{\prime} b}^{i}$ is a state extension of $\rho_{a b}^{i}$ for all i. Without loss of generality, suppose $\rho_{a a^{\prime} b}^{i}$ is the optimal state extension of $\rho_{a b}^{i}$ for each $i$, and $\sigma_{i}^{\star}$ is the corresponding closest classical-quantum state. Then, we have

$$
\begin{aligned}
& \sum_{i} p_{i} \mathcal{E}_{B}^{a}\left(\rho_{a b}^{i}\right)=\sum_{i} p_{i} d_{B}^{2}\left(\rho_{a a^{\prime} b}^{i}, \sigma_{i}^{\star}\right) \\
= & \sum_{i} p_{i} d_{B}^{2}\left(\rho_{a a^{\prime} b}^{i} \otimes|i\rangle_{a^{\prime \prime}}\left\langle i\left|, \sigma_{i}^{\star} \otimes\right| i\right\rangle_{a^{\prime \prime}}\langle i|\right) \\
\geq & d_{B}^{2}\left(\sum_{i} p_{i} \rho_{a a^{\prime} b}^{i} \otimes|i\rangle_{a^{\prime \prime}}\left\langle i\left|, \sum_{i} p_{i} \sigma_{i}^{\star} \otimes\right| i\right\rangle_{a^{\prime \prime}}\langle i|\right) \\
\geq & \hat{D}_{B}\left(\sum_{i} p_{i} \rho_{a a^{\prime} b}^{i} \otimes|i\rangle_{a^{\prime \prime}}\langle i|\right) \\
\geq & \hat{\mathcal{E}}_{B}\left(\sum_{i} p_{i} \rho_{a b}^{i}\right),
\end{aligned}
$$

where the first inequality follows from the joint convexity of $d_{B}^{2}$ and the second inequality is based on the definition of $\hat{D}_{B}$ and the fact that $\sum_{i} p_{i} \sigma_{i}^{\star} \otimes|i\rangle_{a^{\prime \prime}}\langle i|$ is a classical-quantum state along the bipartition $a a^{\prime} a^{\prime \prime}: b$. The last inequality follows because $\sum_{i} p_{i} \rho_{a a^{\prime} b}^{i} \otimes|i\rangle_{a^{\prime \prime}}\langle i|$ is a state extension of $\sum_{i} p_{i} \rho_{a b}^{i}$.

\section{B. Proof of Theorem 4}

Here we restate the claim to be proved. That is, for each $\rho_{a b} \in D(\mathcal{H})$

$$
E_{B}\left(\rho_{a b}\right) \leq \hat{\mathcal{E}}_{B}\left(\rho_{a b}\right) \leq E_{B}^{c r}\left(\rho_{a b}\right) .
$$


Proof. Suppose $\rho_{a a^{\prime} b}^{\star}$ is the optimal state extensions of $\rho_{a b}$ and $\sigma^{\star}$ is the corresponding closest classical-quantum state. Then

$$
\hat{\mathcal{E}}_{B}\left(\rho_{a b}\right)=d_{B}^{2}\left(\rho_{a a^{\prime} b}^{\star}, \sigma^{\star}\right) \geq d_{B}^{2}\left(\rho_{a b}, t r_{a^{\prime}} \sigma^{\star}\right) \geq E_{B}\left(\rho_{a b}\right) .
$$

The first " $\geq "$ is the result of the contractibility of Bures distance and the second " $\geq$ " is because $t r_{a^{\prime}} \sigma^{\star}$ is a separable state. In fact, if $\sigma^{\star}=\sum_{i} p_{i}\left|\alpha_{i}\right\rangle_{a a^{\prime}}\left\langle\alpha_{i}\right| \otimes \rho_{i}$, where $\left|\alpha_{i}\right\rangle_{a a^{\prime}}=\sum_{k} \lambda_{k}^{i}\left|x_{k}^{i}\right\rangle_{a}\left|y_{k}^{i}\right\rangle_{a^{\prime}}$, then tracing out the subsystem $a^{\prime}$ will lead to a decomposition of the form $t r_{a^{\prime}} \sigma^{\star}=$ $\sum_{i, k} p_{i} \lambda_{k}^{i}\left|x_{k}^{i}\right\rangle_{a}\left\langle x_{k}^{i}\right| \otimes \rho_{i}$, that must be a separable state.

In particular, let us consider the pure state case. Suppose $|\psi\rangle=\sum_{i} \sqrt{\lambda_{i}}\left|x_{i}\right\rangle_{a}\left|y_{i}\right\rangle_{b}$ with $\lambda_{1} \geq \ldots \geq \lambda_{n}$. Then

$$
\begin{gathered}
\hat{\mathcal{E}}_{B}(|\psi\rangle) \leq \min _{\sigma_{a a^{\prime} b} \in \mathcal{C} \mathcal{Q}} d_{B}^{2}\left(|\psi\rangle_{a b}\langle\psi|\otimes| u\rangle_{a^{\prime}}\langle u|, \sigma_{a a^{\prime} b}\right) \\
\leq d_{B}^{2}\left(|\psi\rangle_{a b}\langle\psi|\otimes| u\rangle_{a^{\prime}}\langle u|,\right. \\
\left.\quad\left|x_{1}, y_{1}\right\rangle_{a b}\left\langle x_{1}, y_{1}|\otimes| u\right\rangle_{a^{\prime}}\langle u|\right) \\
=E_{B}(|\psi\rangle) .
\end{gathered}
$$

Combining the above two resulta, one has $\hat{\mathcal{E}}_{B}(|\psi\rangle)=$ $E_{B}(|\psi\rangle)$ for any pure state $|\psi\rangle$.

On the other hand, for any mixed state with pure state decomposition $\rho_{a b}=\sum_{i} p_{i}\left|\psi_{i}\right\rangle_{a b}\left\langle\psi_{i}\right|$, Theorem 1 tells us that

$$
\hat{\mathcal{E}}_{B}\left(\rho_{a b}\right) \leq \sum_{i} p_{i} \hat{\mathcal{E}}_{B}\left(\left|\psi_{i}\right\rangle\right)=\sum_{i} p_{i} E_{B}\left(\left|\psi_{i}\right\rangle\right) .
$$

Taking the minimum over all pure state decompositions,

$$
\hat{\mathcal{E}}_{B}\left(\rho_{a b}\right) \leq E_{B}^{c r}\left(\rho_{a b}\right) .
$$




\begin{tabular}{|c|c|}
\hline Symbol & Explanation \\
\hline $\mathcal{S}$ & set of separable states \\
\hline $\mathcal{C Q}$ & set of classical-quantum states \\
\hline $\mathcal{C C}$ & set of classical-classical states \\
\hline$(\hat{D}) \tilde{D}$ & (a)symmetric discord \\
\hline$\left(\hat{D}_{B}\right) \tilde{D}_{B}$ & (a)symmetric Bures distance of discord \\
\hline$d_{B}$ & Bures distance \\
\hline$E_{d}$ & geometric entanglement \\
\hline$E_{B}$ & Bures distance of entanglement \\
\hline$E_{B}^{c r}$ & convex roof of Bures distance of entanglement \\
\hline$(\hat{\mathcal{E}}) \tilde{\mathcal{E}}$ & minimal (a)symmetric DSE \\
\hline$\left(\hat{\mathcal{E}}_{B}\right) \tilde{\mathcal{E}}_{B}$ & minimal (a)symmetric Bures distance of DSE \\
\hline$\left(\hat{\mathcal{E}}_{d}\right) \tilde{\mathcal{E}}_{d}$ & minimal (a)symmetric DSE \\
\hline$\left(\hat{\mathcal{E}}_{d}^{\prime}\right) \tilde{\mathcal{E}}_{d}^{\prime}$ & minimal (a)symmetric MIDSE \\
\hline$\left(\breve{\mathcal{E}}_{d}\right) \breve{\mathcal{E}}_{d}$ & minimal partial trace of (a)symmetric DSE \\
\hline$\left(\check{\mathcal{E}}_{d}^{\prime}\right) \breve{\mathcal{E}}_{d}^{\prime}$ & minimal partial trace of (a)symmetric MIDSE \\
\hline
\end{tabular}

TABLE I. Symbols and their meanings. Here DSE stands for discord over state extensions and MIDSE is for measurement-induced geometric discord over state extensions. 